\title{
Description of the sagittal jaw relation in cephalometric analysis - a review of literature
}

\author{
Maciej Jedliński ${ }^{1, A 凶}$, Joanna Janiszewska-Olszowska², B, Katarzyna Grocholewicz², c \\ ${ }^{1}$ Pomeranian Medical University in Szczecin, Undergraduate student Scientific Society at Department of Interdisciplinary Dentistry, Powstańców Wlkp. 72, 70-111 \\ Szczecin, Poland \\ ${ }_{2}^{2}$ Pomeranian Medical University in Szczecin, Department of Interdisciplinary Dentistry, Powstańców Wlkp. 72, 70-111 Szczecin, Poland \\ ${ }^{\text {A }}$ ORCID: 0000-0003-3446-6119; ${ }^{\text {B } O R C I D: ~ 0000-0003-4374-2568 ; ~}{ }^{\mathrm{C}}$ ORCID: 0000-0002-1468-4664 \\ $\bowtie$ maciej.jedlinski@hotmail.com
}

\begin{abstract}
Introduction: Lateral cephalometry is commonly used to analyse craniofacial morphology, soft tissue profile and the direction of facial growth in an attempt to predict the possibilities and limits of orthodontic therapy.

The aim of the study was to present the most frequently used cephalometric measurements to assess the skeletal class on a lateral cephalometric headfilm.

Materials and methods: A Pubmed search was undertaken to systematize information on ANB angle, WITS appraisal, APDI and Harvold measurements. The keywords used were "cephalometry AND evaluation of sagittal malocclusion AND reference lines AND accuracy OR ANB angle OR WITS appraisal OR
\end{abstract}

APDI". A manual search was performed from the reference lists of studies found in order to identify and include pioneer studies. Results: A total number of 1258 records were found and finally 23 studies were included in the review. Four of them were descriptive, the other 19 were randomized control trials.

Conclusions: 1. ANB angle cannot be used as the only indicator of sagittal skeletal discrepancy. 2. WITS appraisal is independent of the variability of cranial base structures, thus may be an important supplement to the diagnosis, although it depends on the variability of the occlusal plane. 3. APDI can reliably distinguish between class I, II and III malocclusions. Keywords: ANB; WITS; AF-BF; AH-BH; APDI.

\section{INTRODUCTION}

Anterior-posterior discrepancies are analysed in relation to the orbital plane. They may result from dentoalveolar or skeletal discrepancies (caused by an altered relation of the maxilla and mandible). Diagnosing the aetiology of a malocclusion is crucial in planning a proper orthodontic treatment. Orthodontic correction of skeletal defects is generally only possible in growing patients. After growth ceases, treatment of skeletal discrepancies relies mainly on dentoalveolar compensation or orthodontics followed by orthognathic surgery. Treatment of dentoalveolar malocclusion can be performed by orthodontic tooth movement at most ages. Thus a comprehensive cephalometric analysis is essential in treatment planning.

Lateral cephalometry is commonly used to analyse craniofacial morphology, soft tissue profile and the direction of facial growth in attempt to predict the possibilities and limits of orthodontic therapy. The 1st scientific report on cephalometry was published in 1922 by Pacini and disseminated in the 1930s [1]. For almost a century, many other cephalometric analyses have been created, which are used to varying degrees and recommended by individual academic centres, the most popular of which are: Steiner's method (Europe), Jarabak (United States, Italy), Segner-Hasund (Poland, Germany), as well as Rickets, Björk, and Arnett. In recent years, Kim's analysis has become popular. It was developed and published by Kim and Vietas in 1974 [2], currently promoted by Professor Sadao Sato, who uses it for planning the MEAW (Multiple Edgewise Arch Wire) treatment.

In orthodontic diagnosis, the skeletal class is used to classify the sagittal position of the maxilla and mandible. Class I reflects a normal sagittal relation, while class II and III relate to discrepancies in the sagittal plane, often associated by malocclusions of several degrees of severity - mild, moderate and severe.

Each cephalometric analysis is based on landmarks and measurements (angular and linear). Cephalometric landmarks can be classed as anatomical or structural. Moreover, skeletal, dental and soft tissue landmarks may be used. Some landmarks and reference lines are commonly used by many of the analyses. Individual measurements have been analysed in a population (studied by the author of the analysis), thus mean population values may be found in literature. The analysis by Segner and Hasund is based on correlations between cephalometric measurements derived from a population study [3]. A special "Harmonie-box" is used to define individual norms and proportions of every patient according to a facial type defined by cephalometric measurements.

The aim of the study was to present the most frequently used cephalometric measurements to assess the skeletal class on a lateral cephalometric headfilm. 


\section{MATERIALS AND METHODS}

A Pubmed search was undertaken to systematize information on ANB angle, WITS appraisal, anteroposterior dysplasia indicator (APDI) and Harvold measurements. The keywords used were "cephalometry AND evaluation of sagittal malocclusion AND reference lines AND accuracy OR ANB angle OR wits appraisal OR APDI". The search was made in English and no time limit was established (last search date was 24.07.2019). The selection criteria were: English language, 2-dimensional lateral cephalometric analysis. Review papers were excluded. All full texts of the selected studies were accessed and analysed. A manual search was performed in the reference lists of studies found in order to identify and include pioneer studies.

\section{RESULTS}

A total number of 1258 records were found. Finally 23 studies were included. Four of those studies described the measurements used to describe the sagittal jaw relation, the other 19 are randomized clinical trials (RCT) comparing the efficiency and diagnostic accuracy of various cephalometric measurements.

\section{DISCUSSION}

Standardization of the cephalogram carried out by Holly Broadbent in 1931 simplified the diagnosis of skeletal disorders [4].

\section{ANB angle}

Various cephalometric measurements are used to assess sagittal skeletal discrepancies. Downs described the angle of plane $\mathrm{AB}$ and the angle of convexity in the assessment of anterior-posterior dysplasia of the jaws. In 1952 Riedel was the 1st to publish a report in which ANB angle was used to assess sagittal discrepancies [5]. This measurement was later popularized by Steiner [6]. This angle is nowadays used in many analyses and calculated as the arithmetic difference between the SNA and SNB angles (Fig. 1). However, there is a divergence in clinical mean values and their interpretation in individual analyses (Tab. 1).
ANB angle did not appear in the original Schwarz analysis, however it is included in the analysis for its diagnostic value. The sagittal relation between the maxillary base and the jaws in Schwarz's analysis is described by the $\mathrm{AB}$ angle formed at the intersection of the $\mathrm{AB}$ and $\mathrm{SpP}$ lines (the plane of the maxillary base), measured externally. The average value is 90 degrees. An obtuse angle is characteristic for a distal position of the alveolus of the mandible in relation to the maxilla, which is present in II class malocclusions, an acute angle indicates a protrusion of the alveolar part of the mandible, characteristic for III class malocclusions. The MM angle between the lines A-Pog and SpP (also measured externally) is on average 90 degrees. A greater MM angle indicates a distal position of the mandible in relation to the maxilla, a lesser angle describes a mandibular protrusion.

In the analysis by Segner and Hasund [3], neutral, mesial and distal relations are distinguished based on ANB angle, however analysing the sagittal relationship is performed in relation to the patient's facial type, as different ANB angle values are considered harmonious for the orthognathic, prognathic or retrognathic face [3]. Thus sagittal harmony is described in an individual manner, and this analysis is determined by

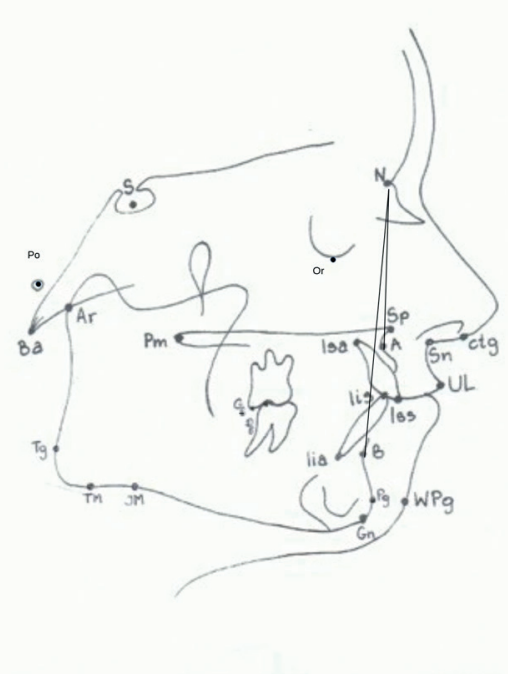

FIGURE 1. ANB angle

TABLE 1. Interpretation of ANB angle in various cephalometric analyses

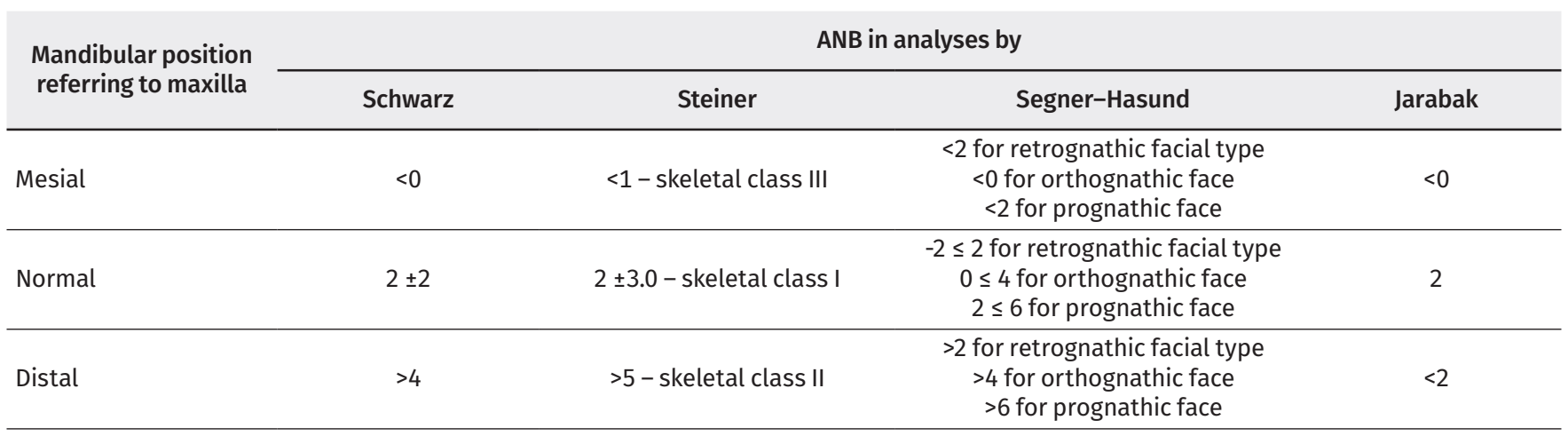


individual cephalometry. A retrognathic maxilla is diagnosed if SNA is below 79 and a retrognathic mandible is diagnosed if SNB is below 77, an orthognathic maxilla and mandible at SNA angle between 79-85 and SNB angle between 77-83, the predicted maxilla and the angle SNA above 85 or the prognosis of the mandible when SNB angle is above 83. In the case of an orthognathic face, both conditions must be met, and in the other 2 only 1 of them is enough [3].

Freeman was one of the first authors to prove that changes in the position of the Nasion can change the value of ANB angle [7]. Later, other authors pointed out that the Nasion point moves forwards and upwards with growth. They found that point A was also not constant, it moved with maxillary growth similarly to the Nasion point.

Taylor stated that ANB angle was not only dependent on the changing position of the Nasion point, but also on the variability of the individual face $[7,8,9]$. Hussels and Nanda summarized, that the factors that affected the value of the ANB angle were:

- the age of the patient. ANB angle has a clear tendency to decrease with age,

- change of the position of the Nasion point in the vertical or horizontal direction,

- upward or downward rotation of the SN line,

- maxillary upward or downward rotation,

- change in the angle between the SN plane and the occlusal plane,

- the degree of facial prognathism [9].

The difference between real and calculated ANB angle is a measure of the size of the skeletal discrepancy. This leads to a new definition of what is meant by skeletal relationships. Classes II and III as ANB angle calculated for the skeletal class I WITS $=0$, can vary widely, being negative or positive [10]. Due to so many factors affecting the value of ANB angle, it can not be treated as an unambiguous indicator enabling the diagnosis of sagittal skeletal relation, despite the undoubted ease of its implementation.

\section{WITS appraisal}

In order to eliminate the influence of the above-mentioned factors, Jacobson proposed to measure the anterior-posterior defect by WITS [10], which relates the skeletal relation to the occlusal plane (Fig. 2). By 1955 Jenkins had chosen a functional occlusal line as a reference for assessing the disharmony of the jaws. He believed that muscle strength works along this plane and is closely related to it. In 1963 he also used the occlusal plane to assess growth direction. However, he was aware that the relationship of the points A and B can be affected by the inclination of the occlusal plane.

In 1976 Jacobson presented the measurement "WITS" (abbreviation for University of Witwatersrand, Johannesburg). Appraisal WITS measurement is obtained by a perpendicular projection of points A and B on the FOL plane (occlusion line). Points A and B should overlap. The longer the distance in the FOL line between points $A$ and $B$, the greater the disorder:

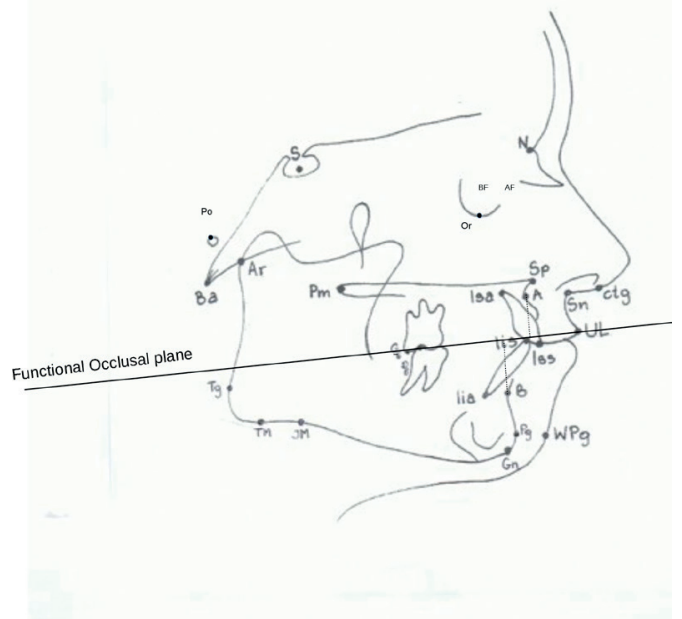

FIGURE 2. WITS appraisal

I skeletal class $0 \pm 2 \mathrm{~mm}$

II skeletal class $>2 \mathrm{~mm}$

III skeletal class $<-2 \mathrm{~mm}$.

WITS describes the sagittal relation between the maxilla and mandible, eliminating the reference points basis on the skull. Among the factors that may cause errors in the interpretation of the disorder, he listed:

- the anterior-posterior position of the Nasion point in relation to the jaw, which can be dependent on many individual factors,

- effect of the rotation of the jaws in relation to cranial reference structures [10].

In everyday practice, this measurement is used in the Steiner analysis with the Kaminek amendment.

Jacobson stated that a high ANB angle in a subject with excellent occlusion may be caused by the anterior position relative to the Nasion point, or by rotation of the jaws in addition to the frontal base of the skull [10]. Moreover, according to this author, the ANB angle was only reliable if the inclination of the mandibular plane was normal. A greater angle of inclination of the mandibular body would indicate a divergent pattern of growth and in many cases, a higher inclination of the anterior skull base that reduces the angle of the SNA and is the cause of a less reliable measurement. Unfortunately, the WITS appraisal has limitations as well, such as a dependence on the variability of an occluded plane.

The high degree of variability in WITS measurements is certain because:

1. eruption of the teeth and development of the teeth can easily affect the occlusal plane,

2. there are difficulties or inaccuracies in identification of the occlusal plane,

3. the inclination of the occlusal plane may vary as a result of vertical movement of the incisors, molars or both during treatment [11]. 
Bishara et al. pointed out, based on studies in patients between 5-25 years old, that ANB angle varies significantly with age, while WITS indicates that the relation between points $A$ and $B$ does not change significantly. ANB and WITS values are significantly correlated, but their value is low. This explains the discrepancies that occur in some cases between the ANB value and the clinical judgment of the clinician. Bishara et al. suggests that in order to obtain a more accurate diagnosis of the sagittal relations of the bone bases, both ANB angle and WITS measurement should be assessed [12].

Simultaneous assessment of the ANB angles and WITS appraisal in cephalometric analysis is used in many orthodontic practices. Many authors emphasize inconsistencies in ANB and WITS angle measurements [12, 13, 14].

According to the research by Ifesanya et al. [13] on the relation between ANB angle and WITS with respect to 3 planes (bisected plane of occlusion, the functional plane of occlusion and a bisected angle maxillary - mandibular MM), it was found that ANB was best moderated by measuring WITS in the plane of the bisector maxilla mandibular MM.

According to Del Santo, the incoherence of ANB angle and WITS results from the angle of inclination of the occlusal plane and vertical features of the craniofacial region [14]. He found inconsistencies in a group of patients with a high inclination angle of the occlusal plane, and a tendency toward cohesion in a group with a low inclination angle of the occlusal plane.

\section{AF-BF}

Many authors aimed at eliminating the inconsistency of ANB and WITS measurements in the assessment of anterolateral relations. Chang, wanting to avoid variations of the occlusal plane, proposed to project points A and B (according to Downs) on the Frankfort horizontal plane, which depends on the variability of the position of Orbital and Porion points [15]. This

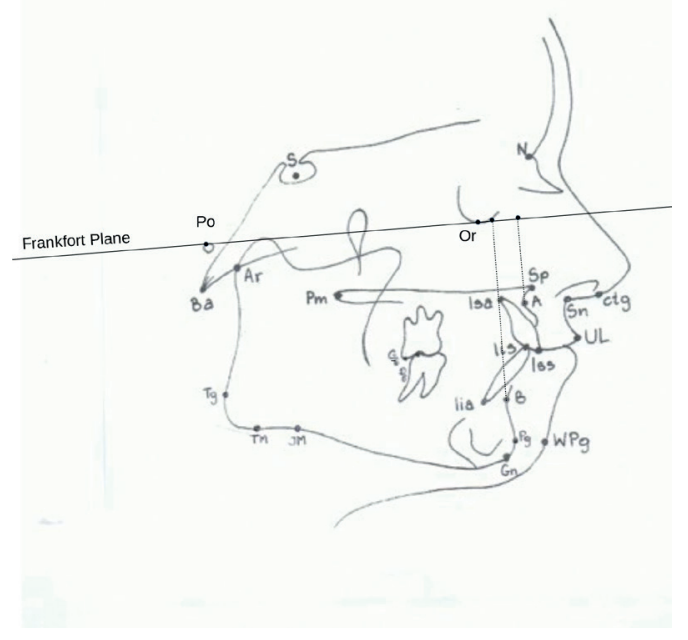

FIGURE 3. AF-BF measurement can be found in literature as AF-BF (Fig. 3). Chang postulated that both skin points are less subject to individual variation than the FOL occlusal plane [15].

\section{The maxillary-mandibular planes angle bisector (MM-AB)}

Hall-Scott proposed a different projection plane for points A and $\mathrm{B}$ to assess the antero-posterior relationship of the maxilla and mandible (Fig. 4) [16]. It is obtained through the construction of a bisector angle based on the plane of the base of the maxilla - designated anterior nasal spine (ANS) and posterior nasal spine (PNS), and the plane of the base of the mandible

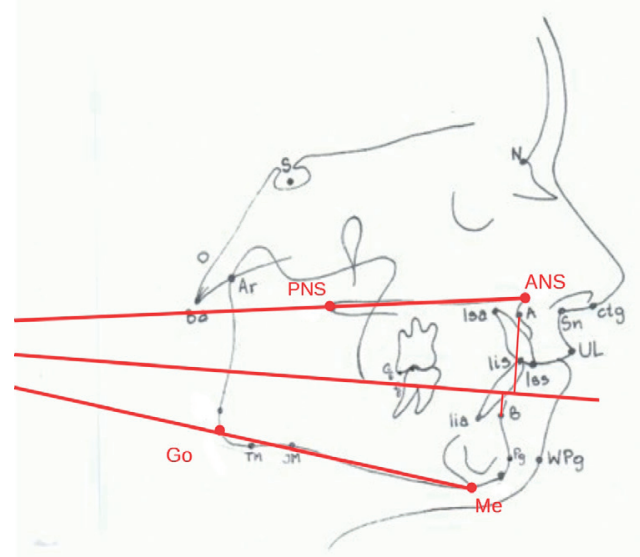

FIGURE 4. The maxillary-mandibular planes angle bisector

passing through the Menton (Me) point and the lowest point in the mandibular angle - Gonion (Go). Hall-Scott believed that due to the bisector position below the occlusal plane, the presence of peaks caused by the teeth and fillings does not affect the accuracy of locating the projection plane, as in the case of FOL [16]. This method, in research by Tiwari et al., showed almost complete accordance with the WITS appraisal for grade I in all age groups, while statistically significant differences were found between ANB and WITS results [17].

\section{AH-BH - measurement on a perfectly perpendicular line}

Other authors have tried to transfer assessment of the horizontal relationship of points $\mathrm{A}$ and $\mathrm{B}$ by determining the plane of their orthogonal projection extracranially, which should ensure independence of the measurement from variations of the occlusal plane. Nagar et al. rebuilt the cephalostat so that the vertical line was on the central sagittal plane defined by 0.009 inch steel wire suspended on a horizontal bar and loaded with $100 \mathrm{~g}$ weight. The cephalogram was performed in the patient's natural head position (NHP) [18]. Each line perpendicular to the true vertical line is a true horizontal line (HOR), which makes 
it possible to evaluate the bone bases on the above-mentioned plane. Referring the assessment of the jaws to the outermost line HOR, he projected points A and B onto it and evaluated the distance between intersection points AH-BH (Fig. 5). The present study showed a better correlation of ANB angle and the horizontal relationship A and B in the HOR (AH-BH) plane than in the case of the WITS assessment.

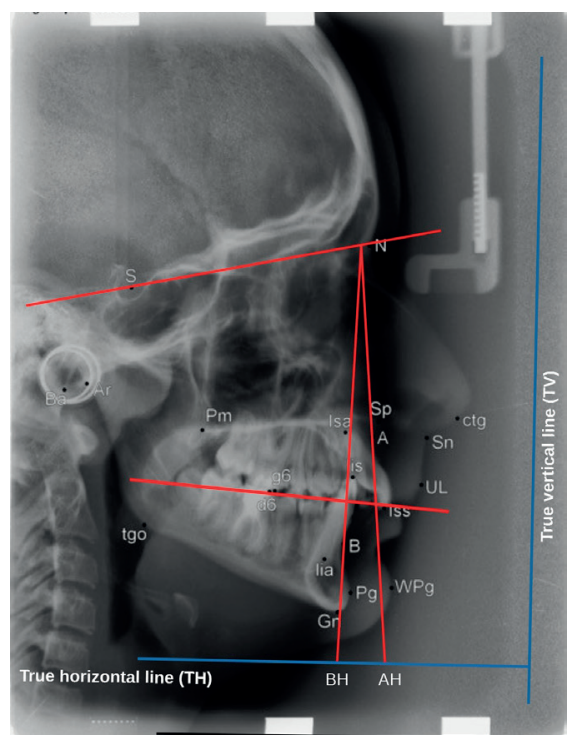

FIGURE 5. AH-BH-measurement on a perfectly perpendicular line

\section{Anteroposterior dysplasia indicator (APDI)}

In Kim's cephalometric analysis, the sagittal relationship of the jaw and mandible is determined by the APDI taking into account the 3 skeletal classes. Kim and Vietas pointed out that changes in the jaws are not caused by one factor, so to properly diagnose a patient, the relationship should be analysed in 3 dimensions [2].

This indicator is the sum of 3 angles (Fig. 6 and 7):

a) the angle between the Frankfurt ( $\mathrm{Po}-\mathrm{O})$ plane and the face plane $(\mathrm{N}-\mathrm{Pg})$ - determining the depth of the face by locating the chin in the horizontal plane and defining the skeletal class as the mandibular position. The average value of this angle is $87 \pm 3$. An open angle occurs in case of a prognathic mandible, an acute angle in the case of a retrognathic mandible,

b) the angle between the $\mathrm{AB}$ plane and facial plane (N-Pg) describes the sagittal relation between the jaws. A negative value of the angle indicates point $A$ is forward of $B$ and defines a class II malocclusion. A positive value indicates point $\mathrm{B}$ is forward of $\mathrm{A}$ and indicates a class III malocclusion. To determine the convexity of the face through the FP AB angle it is necessary to know the angle Frankfort Horizontal (FH) - Facial plane (FP). If the mandible is prognathic, the FP AB angle is sharp with a tendency to positive values. If the mandible is retrognathic and convexity is increased, the angle should be open, indicating a tendency to class II malocclusion, c) the angle between the Frankfort (Po-O) plane and the plane of the palate (ANS-PNS) - it should have a value of 2 degrees. A negative angle indicates that the palate is inclined upwards and forwards, which may or may not be related to a II class malocclusion, whereas a positive angle always means a class III malocclusion.

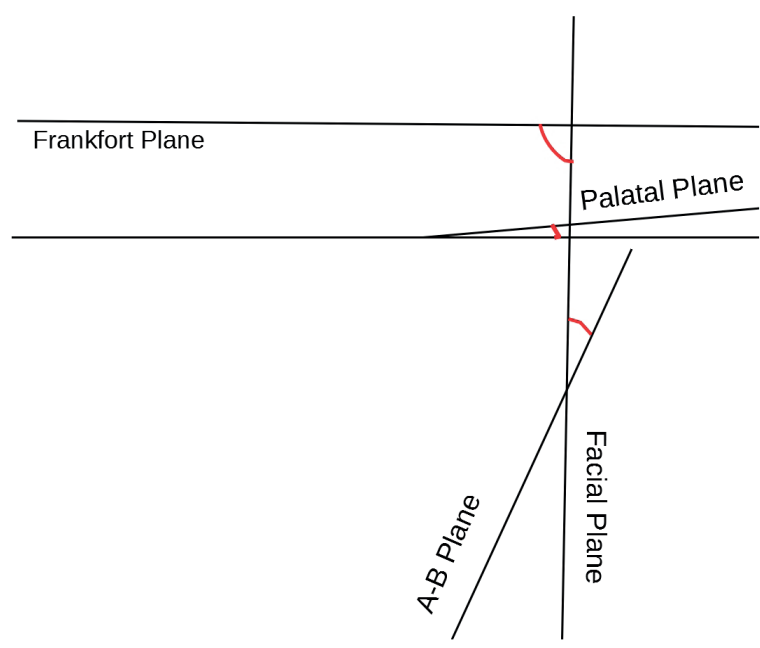

FIGURE 6. Angles forming anteroposterior dysplasia indicator

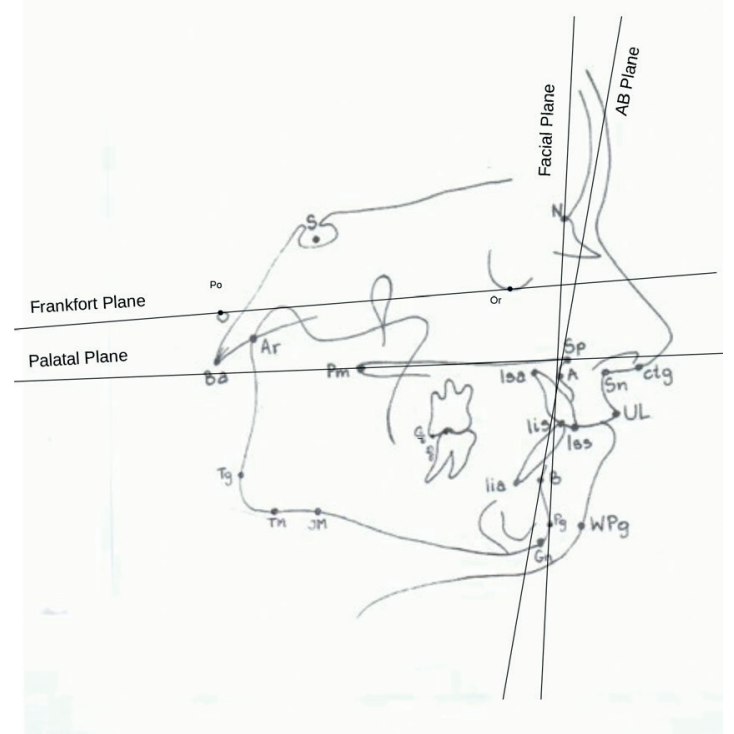

FIGURE 7. Anteroposterior dysplasia indicator plotted on the outline of a cephalometric study

Kim and Vietas suggested a measurement pattern, an anterior-posterior dysplasia index, which is a resultant reading calculated from the face angle plus or minus the angle of plane 
A-B, and again plus or minus the angle of the palatal plane [2]. The sum of the angles should be 81.4 degrees \pm 3.7 . This is a calculation method, not a direct evaluation.

In the study by Oktay, APDI was indicated as the measurement with the smallest ratio of variation among the subjects, whereas, WITS and ANB had larger index variations [19]. This means that APDI is the most reliable indicator. It is negatively correlated to the length of the SN line.

Assessment of the reliability of APDI was carried out by a team from the University of Karachi in Pakistan on a group of 90 patients [20]. The study material consisted of plaster models and lateral cephalograms, divided into 3 groups according to the Angle classification. The APDI measurements were subjected to mathematical analysis (ROC Curve) to check diagnostic reliability. In $88 \%$ of cases, accordance was confirmed with Angle's diagnosis. According to the authors, APDI can reliably distinguish between class I, II and III discrepancies.

Chen et al. checked whether APDI and ODI could be used for the assessment of anterolateral and vertical jaw development in patients with a cleft palate [21]. Instead of the palatal plane they created a substitute plane - PP. Anteroposterior dysplasia indicator and ODI estimated on the basis of the replacement plane of the palate could be used as important criteria for assessing the vertical and sagittal development of the jaw, especially in patients with a cleft palate.

\section{Harvold's index}

Another interesting and not widely used method of analysing the sagittal jaw relationship are tables published in 1955 by Harvold. The length of the maxilla is determined from the distance between the temporomandibular joint (TMJ) to the ANS. The length of the mandible is measured from TMJ to the gnathion point (Gn). The height of the lower part of the face is measured from the point of the ANS to Me - Figure 8.

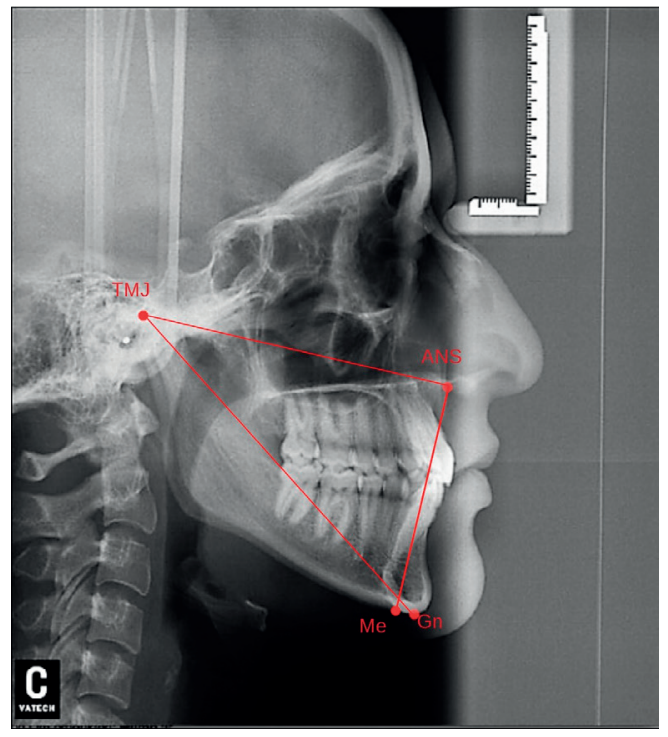

FIGURE 8. Measurements by Harvold
These measurements do not depend on the inclination of the cranial base nor the occlusal plane. Diagnosis of the intermaxillary sagittal relation is made only based on the maxillary and mandibular lengths. These values are compared with the tables developed by the author. This analysis determines which of the jaws is characterized by altered development. In English language literature, no study could be found comparing the concordance of this indicator with other available methods. However, it is considered a good indicator showing the dynamics of development in various faces. This common analysis is used as part of McNamara's cephalometric analysis. In literature, it is often used to compare the size of the face between different races, and within the population of a given country [22].

Cephalometric measurements and calculations may be affected by errors and factors, such as the quality of the radiogram, the physician's interpretation of the chart and the method of forecasting indicators and landmarks. Regardless of the type of analysis used, it is worth considering the purchase of professional cephalometric analysis software in order to standardize and make comparisons more comparable [23].

\section{CONCLUSIONS}

1. ANB angle cannot, despite the ease of measurement, be used as the only indicator to diagnose sagittal skeletal discrepancies.

2. WITS appraisal is an important supplement to the diagnosis of the sagittal relationship of the jaws. It is independent of the variability of cranial base structures, but depends on the variability of the occlusal plane.

3. Due to its high variability, the occlusal plane should be replaced with extracranial planes to obtain accurate and repeatable measurements.

4. APDI, despite the difficulties of implementation, can reliably distinguish between class I, II and III discrepancies.

\section{REFERENCES}

1. Pacini AJ. Roentgen ray anthropometry of the skull. J Radiol 1922;3:230-8.

2. Kim YH, Vietas JJ. Anteroposterior dysplasia indicator: an adjunct to cephalometric differential diagnosis. Am J Orthod 1978;73(6):619-33.

3. Segner D, Hasund A. Individualisierte Kephalometrie. Hamburg: Dietmar Segner Verlag und Vertrieb; 1991.

4. Holly Broadbent B. A new x-ray technique and its application to orthodontia. Angle Orthod 1931:1(2):45-66.

5. Riedel RA. The relation of maxillary structures to cranium in malocclusion and in normal occlusion. Angle Orthod 1952;22(3):142-5.

6. Steiner CC. Cephalometrics for you and me. Am J Orthod 1953;39(10):729-55.

7. Freeman RS. Adjusting A-N-B angles to reflect the effect of maxillary position. Angle Orthod 1981;51(2):162-71.

8. Taylor CM. Changes in the relationship of nasion, point $\mathrm{A}$, point $\mathrm{B}$, and the effect upon ANB. Am J Orthod 1969;56(2):143-63.

9. Hussels W, Nanda RS. Analysis of factors affecting angle ANB. Am J Orthod 1984;85(5):411-23.

10. Jacobson A. Application of the "Wits" appraisal. Am J Orthod 1976;70(2):179-89. 
11. Demisch A, Gebauer U, Fela W. Comparison of the three cephalometric measurements of sagittal jaw relationship and AB-Occlusal angle ANB, wits appraisal and AB/occlusal angle. Trans Eur Orthod Soc 1977:269-81.

12. Bishara SE, Fahl JA, Peterson LC. Longitudinal changes in the ANB angle and Wits appraisal: clinical implications. Am J Orthod 1983;84(2):133-9.

13. Ifesanya JU, Adeyemi AT, Otuyemi OD. The wits appraisal using three reference planes and its interaction with the ANB angle among a sub-set of Nigerians. Afr J Med Med Sci 2014;43(3):225-30.

14. Del Santo M Jr. Influence of occlusal plane inclination on ANB and Wits assessments of anteroposterior jaw relationships. Am J Orthod Dentofacial Orthop 2006;129(5):641-8.

15. Chang HP. Assessment of anteroposterior jaw relationship. Am J Orthod Dentofacial Orthop 1987;92(2):117-22.

16. Hall-Scott J. The maxillary-mandibular planes angle (MM degrees) bisector: A new reference plane for anteroposterior measurement of the dental bases. Am J Orthod Dentofacial Orthop 1994;105(6):583-91.

17. Tiwari R, Shyagali TR, Gupta A, Joshi R, Tiwari A, Sen P. Predictability and reliability of different anterio-posterior skeletal discrepancy indicators in different age groups - a cephalometric study. J Clin Diagn Res 2016;10(9):ZC80-ZC84.
18. Nagar S, Nagar R, Raghav P. Why WITS? Why not a way beyond? Contemp Clin Dent 2014;5(4):518-23.

19. Oktay H. A comparison of ANB, WITS, AF-BF, and APDI measurements. Am J Orthod Dentofacial Orthop 1991;99(2):122-8.

20. Fatima F, Fida M, Shaikh A. Reliability of overbite depth indicator (ODI) and anteroposterior dysplasia indicator (APDI) in the assessment of different vertical and sagittal dental malocclusions: a receiver operating characteristic (ROC) analysis. Dental Press J Orthod 2016;21(5):75-81.

21. Chen ZQ, Qian YF, Shen G, Wang GM. APDI and ODI estimated from substitute palate plane. Shanghai Kou Qiang Yi Xue 2004;13(1):13-6.

22. Lara-Carillo E, Kubodera-Ito T, Gonzalez-Lopez B, Montiel-Bastida N, Esquivel-Pereyra G. Cephalometric norms according to the Harvold's analysis. Int J Odontostomat 2009;3(1):33-9.

23. Nouri M, Hamidiaval S, Akbarzadeh Baghban A, Basafa M, Fahim M. Efficacy of a newly designed cephalometric analysis Software for McNamara analysis in comparison with Dolphin Software. J Dent (Tehran) 2015;12(1):60-9. 